\title{
An open-label, prospective study to evaluate social function and overall improvement of extended-release paliperidone treatment in Thai schizophrenia patients
}

\author{
This article was published in the following Dove Press journal: \\ Neuropsychiatric Disease and Treatment \\ 19 August 2013 \\ Number of times this article has been viewed
}

\author{
Chawanun Charnsil \\ Salinee Vongpanich ${ }^{2}$ \\ 'Department of Psychiatry, Chiang \\ Mai University, Chiang Mai, Thailand; \\ ${ }^{2}$ Medical Affairs Department, Janssen- \\ Cilag, Bangkok, Thailand
}

\begin{abstract}
Background: Previous studies with extended-release (ER) paliperidone have reported an effective outcome in terms of personal and social functioning improvement and also reported schizophrenia symptom improvement. The main objectives of this study were to further investigate improvements in symptom control and social functioning of paliperidone ER and acknowledge the safety profile of paliperidone ER in Thai patients with schizophrenia.
\end{abstract}

Patients and methods: Patients with schizophrenia according to Diagnostic and Statistical Manual of Mental Disorders criteria were allowed flexible 3-12 mg/day dosing during the 10 -week study duration. Patients were interviewed and assessed in social functioning using the Personal and Social Performance (PSP) scale. Patients were also rated on overall severity of illness using the Clinical and Global Impressions - Severity (CGI-S) scale.

Results: In total, 40 patients were enrolled, $80 \%$ of enrolled patients $(n=32)$ completed the 10 -week study period. Thirty-eight eligible patients were included in the intention-to-treat analysis set (male $39.5 \%$, female $60.5 \%$ ). One patient was lost to follow-up without postbaselineefficacy measurements. Another patient was terminated early due to a change in diagnosis during the trial. Statistically significant improvements from baseline in PSP total score were observed at all time points. Clinically relevant improvement in PSP (increase of at least one 10 -point category) was observed in $47.40 \%$ of patients at end point. Improvement in CGI-S was observed at end point $(P<0.001)$. The mean reduction \pm standard deviation at end point in CGI-S was $0.8 \pm 1.04$ (95\% confidence interval $0.48-1.16$ ). The most commonly reported adverse events ( $\geq 5 \%$ of patients) were daytime drowsiness $(15 \%)$ and headache $(15 \%)$. Three subjects $(7.5 \%)$ discontinued due to adverse events.

Conclusion: This study suggests that paliperidone ER is well tolerated in Thai patients with schizophrenia. Paliperidone ER showed improvement in schizophrenic symptom control and social functioning.

Keywords: schizophrenia, social function, paliperidone

\section{Introduction}

Schizophrenia is a severe mental illness affecting about 24 million people worldwide (seven per 1,000 of the adult population). ${ }^{1}$ A person with this disorder has difficulty in differentiating between real and unreal experiences, thinking logically, having normal emotional responses to others, and behaving normally in social situations. ${ }^{1}$ Schizophrenia is also associated with multifaceted cognitive deficits, most prominently in the area of attention, memory, and executive functioning, as well as social cognition..$^{2-5}$ The Diagnostic and Statistical Manual of Mental Disorders, fourth edition,
Correspondence: Chawanun Charnsil Department of Psychiatry, Faculty of Medicine, Chiang Mai University, 239 Huay Kaew Road, Muang,

Chiang Mai 50200, Thailand

Tel +6653945422

Fax +66 $53894 \quad 123$

Email charnsil.research@yahoo.com 
text revision (DSM-IV-TR) lists social/occupational dysfunction as two of six diagnostic criteria for schizophrenia. The description of social functioning includes three domains: work/academic, interpersonal relations, and self-care.

Paliperidone has been developed as a therapeutic agent for the treatment of schizophrenia by Johnson \& Johnson Pharmaceutical Research and Development. It is an active metabolite of risperidone. Unlike other antipsychotic drugs, paliperidone has been developed with an extended-release (ER) formulation to deliver orally administered drug at a relatively constant rate over a 24 -hour period. This minimizes the risk of orthostatic hypotension, and may lead to better efficacy and tolerability of the drug.

Many previous studies have reported the effective outcome of paliperidone ER in terms of schizophrenia symptoms and personal and social functioning improvement. These studies also showed good tolerability among schizophrenia patients. $^{6-9}$

In March 2008, the Thailand Food and Drug Administration approved the market authorization of paliperidone ER. While paliperidone ER has been proven to be safe and efficacious in previous trials, this study is being conducted to further investigate improvements in symptom control and social functioning and to assess safety among the Thai population.

\section{Materials and methods Methods}

This open-label, prospective study was conducted between August 2009 and September 2010 in two centers (Maharaj Nakorn Chiang Mai Hospital and Songklanagarind Hospital) in accordance with current guidelines of the institution regulation and the International Conference on Harmonisation of Technical Requirements for Registration of Pharmaceuticals for Human Use Good Clinical Practice guidelines. The independent ethics committee or institutional review board at each study site approved the protocol. Each participant provided written informed consent before study intervention.

\section{Study populations}

Eligible patients were at least 18 years old and had been diagnosed with schizophrenia according to DSM-IV criteria. Patients were unsatisfied with previous treatment and had previously or were currently on oral atypical antipsychotics.

Exclusion criteria were serious unstable medical condition, including recent and present clinically relevant laboratory abnormalities; history of or current tardive dyskinesia symptoms; and history of neuroleptic malignant syndrome. Pregnancy, breastfeeding patients, and female patients with childbearing potential without adequate contraception were not allowed in this study. Patients with known hypersensitivity to paliperidone ER or risperidone, participants in an investigational drug trial within 30 days prior to selection, and those previously or currently exposed to paliperidone ER were also excluded.

\section{Treatment}

Eligible patients were initiated with paliperidone ER without titration at a dose intended to be effective, selected by the treating physician. In general, treatment was recommended to be started using $6 \mathrm{mg}$ paliperidone ER daily. Flexible maintenance dosing with paliperidone ER 3-12 mg/day was allowed throughout the study period, with dose adjustment indicated by the investigator's assessment based on individual clinical response and tolerance. Concomitant therapy that was permitted included anticholinergic, benzodiazepine, and antidepressant medications.

\section{Assessment}

All investigators were trained on Personal and Social Performance (PSP) scale and Clinical and Global Impressions - Severity (CGI-S) scale rating using standardized training. Interrater reliability was not ascertained.

\section{Efficacy}

At first visit (screening/start of treatment), the patients' demographic data, medical history, previous and current therapy, weight, and overall eligibility were recorded. All time-point analyses employed a last-observation-carriedforward approach for those patients with missing data.

The primary outcome was improvement in social functioning measured by change in PSP scale score. This scale is based on DSM IV-TR social and occupational functioning. It provides the clinician a rating on a 100-point scale, where a higher score represents better personal and social functioning. The PSP scale examines different aspects of function, with the composite score based on ratings in four important domains: (1) socially useful activities, including work and study; (2) personal and social relationships; (3) self care; and (4) disturbing and aggressive behaviors. The PSP scale has been validated in acute and stable patients with schizophrenia. ${ }^{10,11}$ An improvement of greater than or equal to one category (classified as a 10-point interval) on the PSP scale is considered a clinically relevant response..$^{10,12}$ 
The secondary outcome was the CGI-S rating scale to determine the severity of a patient's psychotic condition on a 7-point scale ranging from 1 (not ill) to 7 (extremely severe). The CGI-S scale permits a global evaluation of the patient's condition at a given time. ${ }^{13}$

Patients were interviewed at baseline (visit 1), week 2 (visit 2), week 6 (visit 3), and week 10 (visit 4/end of study) using the PSP scale. Patients were also rated for overall severity of illness at baseline (visit 1) and week 10 (visit 4/ end of study) using the CGI-S scale.

Patients were asked to evaluate their satisfaction with the antipsychotic treatment that they were taking at baseline (visit 1) and end of study (visit 4) on a 5-point Likert scale ranging from "very poor" to "very good." Patients were also asked to rate their quality of sleep and daytime drowsiness at all visits using the visual analog scale. The rating scale for quality of sleep ranged from "very badly" to "very well." Whereas, rating scale for daytime drowsiness was ranged from "not at all" to "all the time."

All efficacy measurements were analyzed for the intention-to-treat population, which was defined as enrolled patients who took at least one dose of study medication and had at least one postbaseline-efficacy evaluation.

\section{Safety}

Safety evaluations were performed on all patients enrolled at every visit throughout the 10 -week study period, and included incidence of adverse events, clinical laboratory, eg, body weight, and vital signs.

\section{Statistical analysis}

Statistical analysis was done using the intention-to-treat principle, and all tests were performed with an alpha level of 0.05 using PASW statistics version 18.0 (IBM, Armonk, NY, USA). Descriptive summaries of demographic variables and baseline characteristics were based on means and standard deviation (SD) for continuous variables, and frequency distributions for categorical variables. Assessment of safety included computation of the incidence of adverse events and presentation in a frequency-distribution table. Paired $t$-tests were performed on PSP scale score, quality of sleep, and daytime drowsiness to determine if there was a significant difference from baseline. Friedman's test was

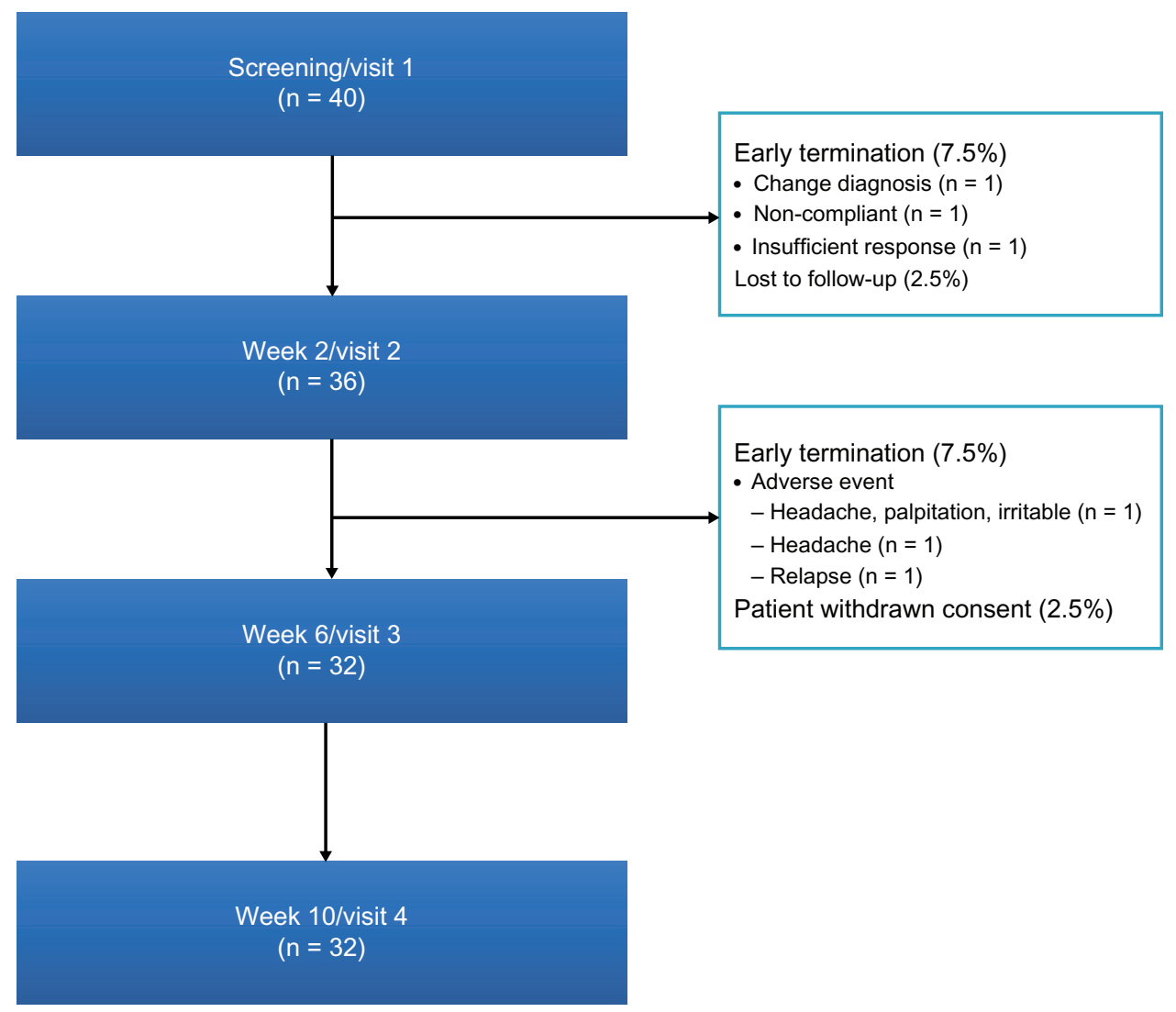

Figure I Patient disposition. 
performed on the CGI-S scale to determine if there was a change in trend from baseline. McNemar's test was done for patient satisfaction. Descriptive analysis was also conducted for CGI-S scale score distribution, patient satisfaction, and score distribution of PSP scale domains. Clinical improvement was achieved with an increase in PSP score of one 10 -point category.

\section{Sample-size calculation}

The sample size to detect the incidence of adverse events from paliperidone $10 \%$ with significant clinical improvement of the schizophrenia subjects depends on the hypothesized acceptable value and the acceptable deviation. Clinical improvement will be revealed with an increase in PSP scale score of 10 points. ${ }^{14}$ With $90 \%$ statistical power and type I error (two-sided) $0.025,35$ subjects were required to prove the hypothesis.

\section{Results}

\section{Demographic and baseline characteristics}

A total of 40 patients were screened and enrolled at two faculty medical hospitals between August 2009 and July 2010 in the study, of which $80 \%(n=32)$ completed the 10 -week study period. A total of 38 eligible patients were included in the intention-to-treat analysis (male $39.5 \%$, female $60.5 \%$ ). One patient was lost to follow-up without postbaseline-efficacy measurement. Another patient was terminated due to early change in diagnosis during the trial (see Figure 1). Demographic and baseline characteristics are shown in Table 1.

Most patients were treated with oral antipsychotics at the time of enrollment. Oral antipsychotic regimens before switching to paliperidone ER were a single atypical

Table I Demographic and baseline characteristic of intent-totreat population $(\mathrm{n}=38)$

\begin{tabular}{lll}
\hline & $\mathbf{n}$ & $\%$ \\
\hline Male & 15 & 39.5 \\
Female & 23 & 60.5 \\
& Mean & SD \\
\hline Age (years) & $4 I . I$ & 12.3 \\
Weight $(\mathrm{kg})$ & 60.6 & $\mathrm{I} 3 . \mathrm{I}$ \\
Height $(\mathrm{m})$ & 1.6 & 0.1 \\
BMI $\left(\mathrm{kg} / \mathrm{m}^{2}\right)$ & 23.7 & 3.84 \\
CGI-S scale & 3.6 & $\mathrm{I} .16$ \\
PSP scale total score (0-I00) & 59.0 & 15.7 \\
Visual analog scale of quality of sleep (0-I) & 0.7 & 0.3 \\
Visual analog scale of daytime drowsiness (0-I) & 0.3 & 0.2 \\
\hline
\end{tabular}

Abbreviations: SD, standard deviation; BMI, body mass index; CGI-S, clinical and global impressions - severity; PSP, personal and social performance.
Table 2 Overall efficacy result $(n=38)$

\begin{tabular}{|c|c|c|c|c|}
\hline Parameters & Baseline & Week 2 & Week 6 & Week 10 \\
\hline PSP scale & 57.47 & 63.24 & 66.00 & 68.82 \\
\hline \multirow[t]{2}{*}{ total score } & (17.976) & $(13.875)$ & (I4.582) & $(14.111)$ \\
\hline & & $P=0.005$ & $P=0.001$ & $P<0.001$ \\
\hline \multirow[t]{2}{*}{ CGI-S scale } & $3.55(1.155)$ & NA & NA & $2.74(1.130)$ \\
\hline & & & & $P<0.001$ \\
\hline Quality & $0.67(0.303)$ & $0.72(0.291)$ & $0.69(0.322)$ & $0.72(0.291)$ \\
\hline of sleep & & $P=0.347$ & $P=0.727$ & $P=0.347$ \\
\hline Daytime & $0.33(0.24 I)$ & $0.3 \mathrm{I}(0.300)$ & $0.23(0.264)$ & $0.22(0.291)$ \\
\hline drowsiness & & $P=0.631$ & $P=0.097$ & $P=0.077$ \\
\hline
\end{tabular}

Abbreviations: CGI-S, clinical and global impressions - severity; PSP, personal and social performance.

antipsychotic (44.7\%), a single typical antipsychotic (15.8\%), two typical antipsychotics (13.2\%), and typical combined with atypical antipsychotics (13.2\%).

At baseline, the $6 \mathrm{mg}$ dose of paliperidone ER was used by most patients $(81.6 \%)$. At the end of the study, $13.2 \%$ of patients reported a dose reduction, while $10.5 \%$ of patients reported a dose increase. At the end point, the mean (SD) dose was 5.84 (1.97) $\mathrm{mg} / \mathrm{day}$, and the distribution of the paliperidone ER dose was $21.1 \%, 65.8 \%, 10.5 \%$, and $2.6 \%$ for $3 \mathrm{mg}, 6 \mathrm{mg}, 9 \mathrm{mg}$, and $12 \mathrm{mg}$, respectively.

\section{Efficacy}

After 10 weeks of paliperidone ER treatment, significant improvements in personal and social functioning were observed at all time points by comparing the mean PSP scale total score with baseline (see Table 2 and Figure 2). In this study, $47.4 \%$ of patients showed a clinically relevant improvement in social functioning (see Table 3). Improvements in difficulty of functioning were observed in all four PSP scale domains (see Figure 3).

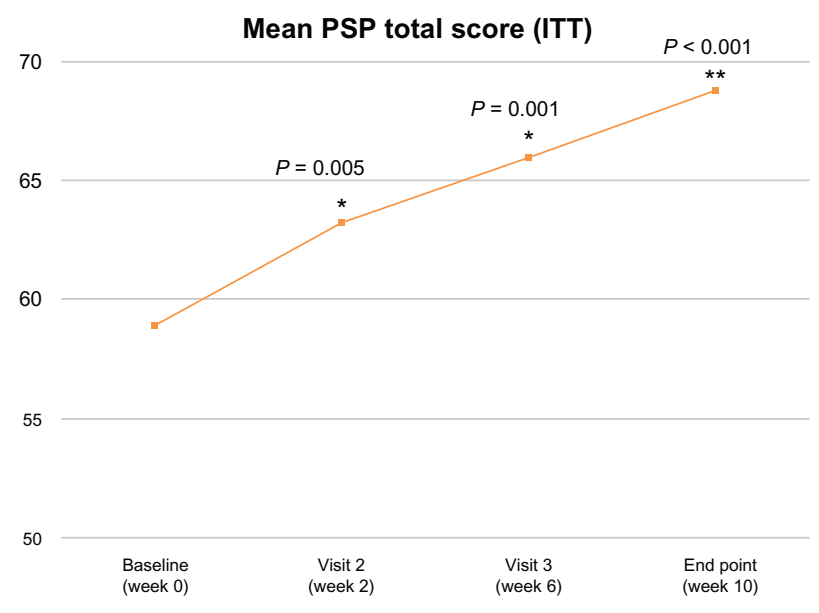

Figure 2 Change in mean PSP scale total score over time last observation carried forward $(n=38)$.

Abbreviations: PSP, personal and social performance; ITT, intention to treat. 
Table 3 Clinically relevant improvement in personal and social performance scale $(n=38)$

\begin{tabular}{lll}
\hline $\begin{array}{l}\text { Change in scale category at } \\
\text { end point (10-point interval) }\end{array}$ & $\mathbf{n}$ & $\%$ \\
\hline$\geq 3$ & 4 & 10.5 \\
2 & 8 & 21.1 \\
1 & 6 & 15.8 \\
0 & 15 & 39.5 \\
-1 & 3 & 7.9 \\
-2 & 2 & 5.3 \\
$\leq-3$ & 0 & 0.0 \\
\hline
\end{tabular}

For the secondary outcome, the CGI-S scale score distribution showed significant reduction in symptom severity at end point. The majority of patients were classified in the "borderline mentally ill" group (34.2\%). After 10 weeks of paliperidone ER treatment, the percentage of patients in "not ill/ borderline mentally ill and mildly ill" groups increases to $71.0 \%$. The patient distribution in the more severe groups "moderately ill", "markedly ill" and "severely ill" at end point is also lower than baseline (23.7\% versus $31.6 \%$, $13.2 \%$ versus $5.3 \%$ and $5.3 \%$ versus $0 \%$, respectively) (see Figure 4). Significant improvement with paliperidone ER was observed at end point in CGI-S scale score at week 10 compared with baseline $(P<0.001)$. The mean \pm SD score at end point was $2.7 \pm 1.13$. This result showed a mean $\pm \mathrm{SD}$ reduction of $0.8 \pm 1.04$ (95\% confidence interval $0.48-1.16$ ) from baseline.
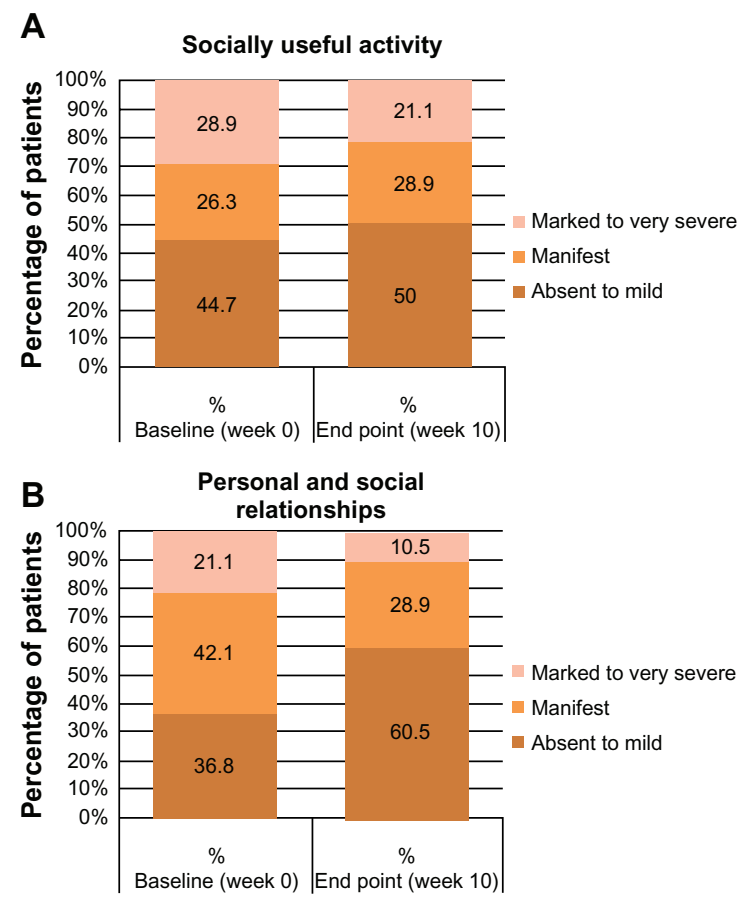

Patient satisfaction toward the current medication changed significantly over the 10 -week period $(P=0.005)$. Patients were more likely to be satisfied with paliperidone ER at end point $(65.80 \%)$ over baseline medications before switching to paliperidone ER (42.10\%) (see Figure 5).

For quality-of-sleep evaluation, all patients were asked how well they had slept in the past 7 nights. There was no significant improvement observed in quality of sleep at end point $(P=0.347)$.

For daytime-drowsiness evaluation, all patients were asked how often they had felt drowsy in the past 7 days. The decreasing number indicated lower daytime drowsiness. The mean \pm SD in daytime-drowsiness improvement from baseline was observed at all time points with mean \pm SD reduction of $0.1 \pm 0.34$ at end point. However, there was no statistically significant difference observed compared to baseline $(P=0.077)$.

\section{Safety and tolerability}

Three subjects $(7.5 \%)$ discontinued the study due to adverse events. One patient experienced three events and reported headache, palpitation, and irritability $(2.5 \%)$. Another patient experienced headache (2.5\%) that caused early withdrawal. A third patient discontinued due to schizophrenia relapse $(2.5 \%)$. Most common adverse events (experienced by at least $5 \%$ of patients) are shown in Table 4. The most commonly reported adverse events
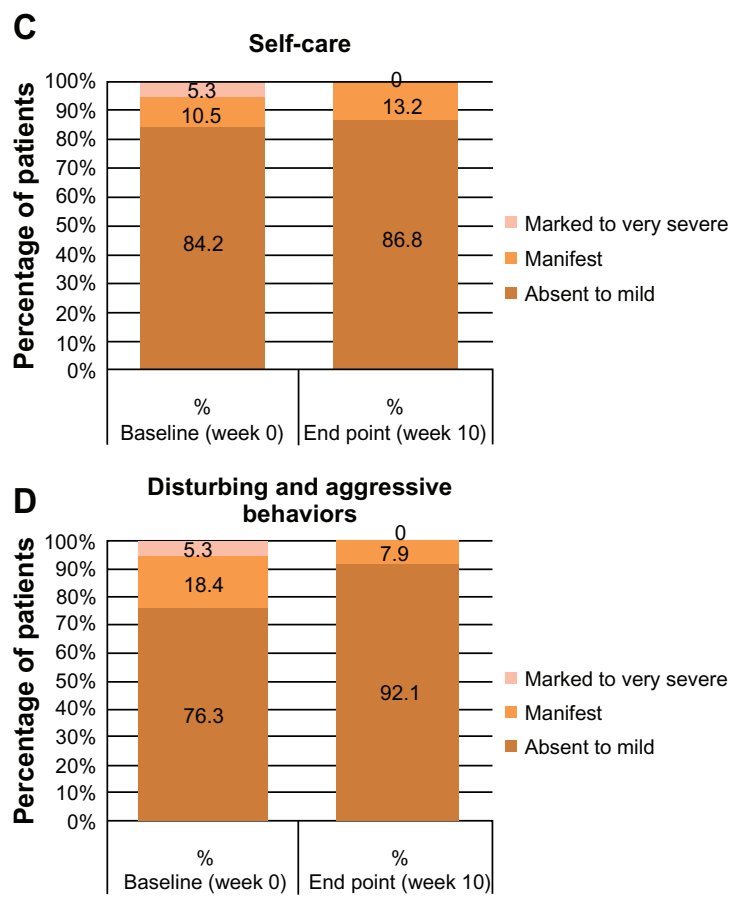

Figure 3 Categories of difficulty in functioning for each Personal and Social Performance scale domain at baseline and end point last observation carried forward ( $\mathrm{n}=38$ ). 


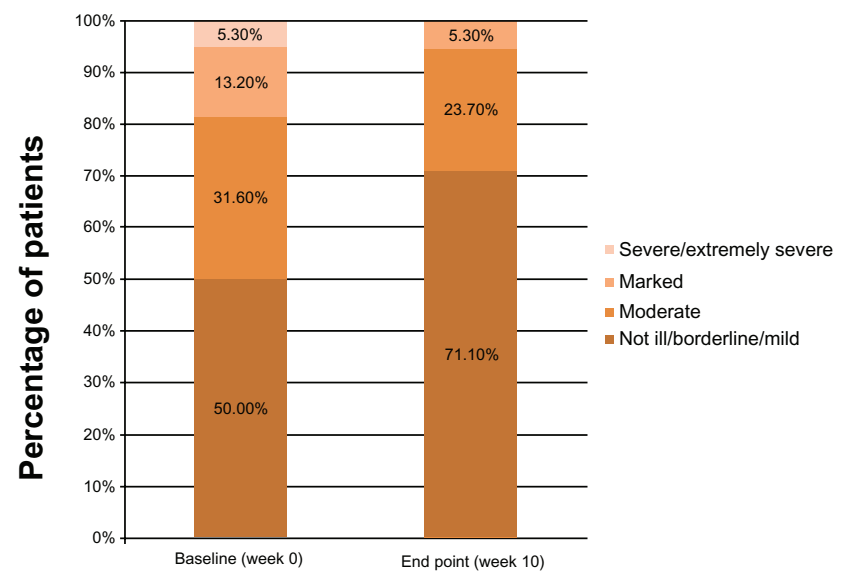

Figure 4 Distribution of Clinical and Global Impressions - Severity scale score $(n=38)$.

( $>5 \%$ of patients) were daytime drowsiness $(15 \%)$, extrapyramidal symptoms (EPSs; 7.5\%), insomnia (7.5\%), and headache $(7.5 \%)$.

Throughout the study period, serious adverse events were reported in one subject $(2.5 \%)$. The subject developed manic symptoms and elevated mood. At that time, the diagnosis was changed to schizoaffective disorder. This subject was hospitalized, and treatment with paliperidone ER was withdrawn. Both manic symptoms and elevated mood were considered doubtfully related to study medication.
Movement disorder-related adverse events were reported by $10 \%$ of patients $(n=4)$. One patient reported akathisia. All EPSrelated adverse events were mild to moderate in severity.

After the 10-week paliperidone ER-treatment period, a weight increase from baseline greater than or equal to $7 \%$ was observed in three subjects $(7.5 \%)$, and mean \pm SD for weight increased $1.09 \pm 0.34 \mathrm{~kg}$ from baseline (see Table 5).

\section{Discussion}

The results from this 10 -week open-label study in patients with schizophrenia who had previously been or were at time of enrollment on oral atypical antipsychotics and were unsatisfied with their treatment demonstrated that paliperidone ER significantly improved social function and overall symptoms. Previous fixed-dose, randomized controlled studies showed a percentage of paliperidone ER-treated patients achieving a clinically meaningful PSP score improvement of between 49\% and 64\%.6,7,15,16 Additionally, another flexible-dose, open-label, prospective study result showed a clinically improvement in PSP score observed in $47.4 \%$ of patients at end point. ${ }^{17}$ The same finding $(47.4 \%)$ was observed in this study. Although most patients did not achieve clinical improvement, short-term interventions limiting their ability to detect meaningful changes in the social functioning of patients may address this result. Similar results indicat-

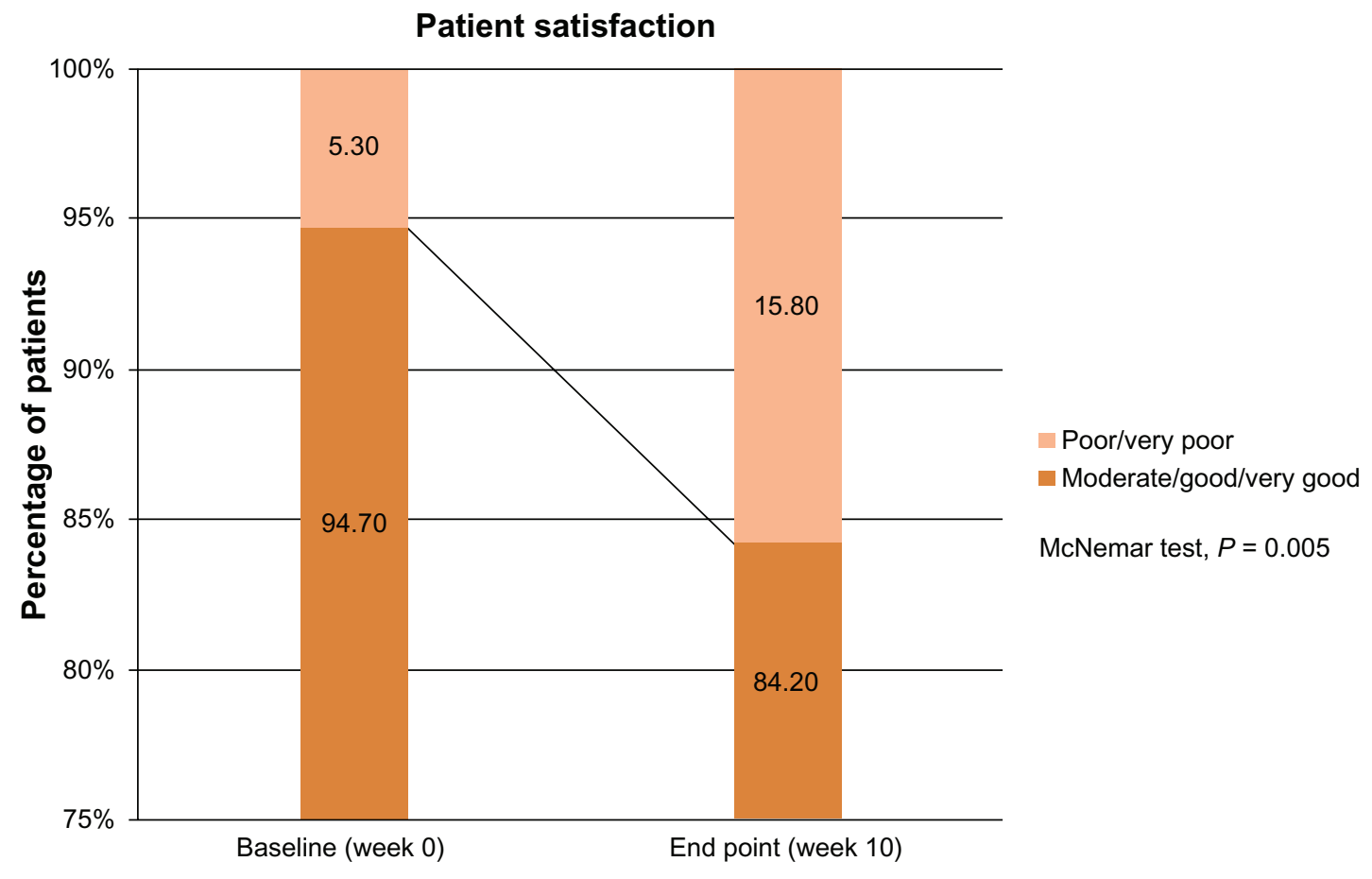

Figure 5 Score distribution of patient satisfaction $(n=38)$. Note: Nobody scored very poorly at all time points. 
Table 4 Adverse events occurring in $\geq 5 \%$ of patients

\begin{tabular}{lll}
\hline Adverse event & $\mathbf{n}$ & $\%$ \\
\hline Daytime drowsiness & 6 & 15.0 \\
EPSs & 3 & 7.5 \\
Headache & 3 & 7.5 \\
Insomnia & 3 & 7.5 \\
Nausea & 2 & 5.0 \\
Vertigo & 2 & 5.0 \\
\hline
\end{tabular}

Abbreviation: EPSs, extrapyramidal symptoms.

ing improvement in social and personal functioning were observed in the high-dose treatment arms of other randomized, placebo-controlled studies (12 mg,15 mg of paliperidone). ${ }^{6,7,15,16}$ The majority of patients in this study received $6 \mathrm{mg}$ of paliperidone ER, suggesting that this is an efficacious and well-tolerated dose for Thai patients. The most frequently used dose of paliperidone ER was $6 \mathrm{mg}$ /day also in a flexibly dosed study in Taiwan. ${ }^{17}$ This showed similar clinical response and safety profile within the Asian population.

Reduction in CGI-S scale score demonstrated that paliperidone ER provided significant improvement of overall psychiatric symptoms. Although the trend was consistent with those from other paliperidone ER studies, ${ }^{6,7,15,16}$ our study showed a lower percentage of patients achieving moderate, good, and very good CGI-S scale categories. This may be explained by the lower baseline severity of schizophrenia symptoms in the patients enrolled in this study compared to other studies.

Paliperidone ER was generally well tolerated throughout the 10 -week study treatment period. No unexpected tolerability finding was observed. The overall discontinuation rate of this study is consistent with other recent published articles. ${ }^{17-19}$ The percentage of patients discontinuing due to adverse events was relatively low $(7.5 \%)$. This result is comparable with Emsley et al (7\%). ${ }^{9}$ There was a patient who experienced serious adverse events, with manic symptoms and mood elevation. The diagnosis of this patient's disorder was changed to schizoaffective disorder, and consequently the subject was withdrawn because of the violation of the inclusion criteria. Both manic symptoms

Table 5 Change in metabolic parameters (baseline to end point) $(n=40)$

\begin{tabular}{lllll}
\hline Metabolic & $\begin{array}{l}\text { Visit I } \\
\text { parameters }\end{array}$ & $\begin{array}{l}\text { Visit 4 } \\
\text { (baseline), } \\
\text { mean (SD) }\end{array}$ & $\begin{array}{l}\text { Change at } \\
\text { mean (SD) }\end{array}$ & $\begin{array}{l}\text { P-value } \\
\text { end point, }\end{array}$ \\
\hline mean (SD) & \\
\hline BMly weight (kg) $\left(\mathrm{kg} / \mathrm{m}^{2}\right)$ & $60.55(\mathrm{I} 3.09)$ & $61.64(\mathrm{I} 3 . \mathrm{II})$ & $\mathrm{I} .09(0.34)$ & 0.003 \\
\hline
\end{tabular}

Abbreviations: SD, standard deviation; BMI, body mass index. and elevated-mood events were considered doubtfully related to study medication. No other serious adverse events were reported. Incidences of other adverse events such as daytime drowsiness, and EPS were comparable with previous studies.

It has been observed that obesity is well recognized in patients with mental illness. In addition, weight gain is likely to occur in patients treated with antipsychotics. ${ }^{20-23}$ In this study, mean weight increase from baseline to week $10(1.09 \pm 0.34 \mathrm{~kg})$ was similar to results reported by Emsley et al $(1.2 \pm 5.16 \mathrm{~kg}){ }^{9}{ }^{9}$ The incidence of patients gaining weight above $7 \%$ from baseline was $7.5 \%$, which is similar to the findings from a meta-analysis of randomized controlled trials $(8 \%){ }^{24}$

Interpreting data from the current study may be limited by the use of an open-label design and lack of a comparator arm. It will be difficult to make conclusions regarding efficacy and safety. In addition, concomitant anticholinergics, benzodiazepine, and antidepressants were allowed, which might have resulted in confounding effects, especially related to adverse events. However, the open-label design with flexible-dose treatment and less strictly prohibited concomitant medications simulates current clinical treatment practice in hospital settings. Other points to be considered include the small number of patients enrolled and the study duration, which might not affect efficacy in long-term functioning. Largescale and long-term investigation in Thai patients may be required to support the social and functioning efficacy of paliperidone ER.

\section{Conclusion}

This study suggests that paliperidone ER is well tolerated in Thai patients with schizophrenia. Paliperidone ER showed significant improvement in schizophrenic symptom control and social functioning. No unexpected tolerability finding was observed. Paliperidone ER appears to be an effective option for schizophrenia treatment in Thailand, and provides social and functioning improvement.

\section{Registration}

This study is registered at ClinicalTrials.gov (NCT01387542). ${ }^{25}$

\section{Acknowledgments}

The authors would like to thank Assistant Professor Arnont Vittayanont, another investigator, from Songklanagarind Hospital. This research was sponsored by Janssen-Cilag Thailand. The poster presentation at the 21st European 
Congress of Psychiatry 2013 was sponsored by the Faculty of Medicine, Chiang Mai University.

\section{Disclosure}

Associate Professor Chawanun Charnsil was a principal investigator for this study and received support to be an advisory board member for Janssen-Cilag Thailand. Salinee Vongpanich is an employee of Janssen-Cilag Thailand.

\section{References}

1. World Health Organization. Mental health: schizophrenia. Available from: http://www.who.int/mental_health/management/schizophrenia/ en. Accessed June 19, 2013.

2. American Psychiatric Association. American Psychiatric Association Practice Guidelines for the Treatment of Psychiatric Disorders: Compendium 2006. Arlington (VA): American Psychiatric Publishing; 2006.

3. Green MF. What are the functional consequences of neurocognitive deficits in schizophrenia? Am J Psychiatry. 1996;153:321-330.

4. Matza LS, Buchanan R, Purdon S, Brewster-Jordan J, Zhao Y, Revicki DA. Measuring changes in functional status among patients with schizophrenia: the link with cognitive impairment. Schizophr Bull. 2006;32:666-678

5. Tollefson GD. Cognitive function in schizophrenic patients. J Clin Psychiatry. 1996;57 Suppl 11:31-39.

6. Davidson M, Emsley R, Kramer M, et al. Efficacy, safety and response of paliperidone extended-release tablets (paliperidone ER): results of a 6-week, randomized, placebo-controlled study. Schizophr Res. 2007;93:117-130.

7. Kane J, Canas F, Kramer M, et al. Treatment of schizophrenia with paliperidone extended-release tablets: a 6-week placebo-controlled trial. Schizophr Res. 2007;90:147-161.

8. Kramer M, Simpson G, Maciulis V, et al. Paliperidone extendedrelease tablets for prevention of symptom recurrence in patients with schizophrenia: a randomized, double-blind, placebo-controlled study. J Clin Psychopharmacol. 2007;27:6-14.

9. Emsley R, Berwaerts J, Eerdekens M, et al. Efficacy and safety of oral paliperidone extended-release tablets in the treatment of schizophrenia: pooled data from three 52-week open-label studies. Int Clin Psychopharmacol. 2008;23:343-356.

10. Nasrallah H, Morosini P, Gagnon DD. Reliability, validity and ability to detect change of the Personal and Social Performance Scale in patients with stable schizophrenia. Psychiatry Res. 2008;161:213-224.

11. Patrick DL, Burns T, Morosini P, et al. Reliability, validity and ability to detect change of the clinician-rated Personal and Social Performance scale in patients with acute symptoms of schizophrenia. Curr Med Res Opin. 2009;25:325-338.
12. Morosini PL, Magliano L, Brambilla L, et al. Development, reliability and acceptability of a new version of the DSM-IV Social and Occupational Functioning Assessment Scale (SOFAS) to assess routine social functioning. Acta Psychiatr Scand. 2000;101:323-329.

13. Berk M, Ng F, Dodd S, et al. The validity of the CGI severity and improvement scales as measures of clinical effectiveness suitable for routine clinical use. J Eval Clin Pract. 2008;14:979-983.

14. Canuso CM, Youssef EA, Bossie CA, Turkoz I, Schreiner A, Simpson GM. Paliperidone extended-release tablets in schizophrenia patient previously treated with risperidone. Int Clin Psychopharmacol. 2008;23:209-215.

15. Marder SR, Kramer M, Ford L, et al. Efficacy and safety of paliperidone extended-release tablets: results of a 6-week, randomized, placebocontrolled study. Biol Psychiatry. 2007;62:1363-1370.

16. Melzer HY, Bobo WV, Nuamab IF, et al. Efficacy and tolerability of oral paliperidone extended-release tablets in the treatment of acute schizophrenia: pooled data from three 6-week, placebo-controlled studies. J Clin Psychiatry. 2008;69:817-829.

17. Huang MW, Yang TT, Ten PR, et al. Effects of paliperidone extended release on the symptoms and functioning of schizophrenia. BMC Clin Pharmacol. 2012;12:1-11.

18. Schmauss M, Jukic V, Siracusano A, et al. Flexible dosing with paliperidone ER in the treatment of patients with acutely exacerbated schizophrenia: results from a single-arm, open-label study. Curr Med Res Opin. 2012;8:1395-1404.

19. Thavichachart N, Kongsakon R, Lo WT, et al. The psychopathological characteristics of treatment discontinuation group in 6-month treatment with paliperidone ER. Int J Clin Pract. 2012;66:969-975.

20. Birt J. Management of weight gain associated with antipsychotics. Ann Clin Psychiatry. 2003;15:49-57.

21. Allison DB, Mentore JL, Heo M, et al. Antipsychotic-induced weight gain: a comprehensive research synthesis. Am J Psychiatry. 1999;156: 1686-1696.

22. Allison DB, Casey DE. Antipsychotic-induced weight gain: a review of the literature. J Clin Psychiatry. 2001;62 Suppl 7:22-31.

23. Allison DB, Fontaine KR, Heo M, et al. The distribution of body mass index among individuals with and without schizophrenia. J Clin Psychiatry. 1999;60:215-220.

24. Harrington CA, English C. Tolerability of paliperidone: a meta-analysis of randomized, controlled trials. Int Clin Psychopharmacol. 2010;25: 334-341.

25. Janssen-Cilag Ltd., Thailand. Safety and Efficacy Study of Paliperidone Extended Release (ER) Among Thai Schizophrenia Participants (PRESENT). Available from: http://clinicaltrials.gov/ct2/show/ NCT01387542.
Neuropsychiatric Disease and Treatment

\section{Publish your work in this journal}

Neuropsychiatric Disease and Treatment is an international, peerreviewed journal of clinical therapeutics and pharmacology focusing on concise rapid reporting of clinical or pre-clinical studies on a range of neuropsychiatric and neurological disorders. This journal is indexed on PubMed Central, the 'PsycINFO' database and CAS.

\section{Dovepress}

The manuscript management system is completely online and includes a very quick and fair peer-review system, which is all easy to use. Visit http://www.dovepress.com/testimonials.php to read real quotes from published authors. 